\title{
Microsatellite Markers and Genetic Diversity in Japanese Apricot (Prunus mume)
}

\author{
Zhi-Hong Gao and Zhi-Jun Shen \\ College of Horticulture, Nanjing Agricultural University, Nanjing 210095, \\ P.R. China
}

\author{
Zhen-Hai Han \\ Institute for Horticultural Plant, China Agricultural University, Beijing \\ 100094, P.R. China
}

\section{Jing-Gui Fang, Yu-Ming Zhang, and Zhen Zhang1 \\ College of Horticulture, Nanjing Agricultural University, Nanjing 210095, P.R. China}

Additional index words. microsatellite, genetic diversity, simple-sequence repeat markers, SSR

\begin{abstract}
Sequencing amplification fragments produced using simple-sequence repeat (SSR) primer pairs pchgms2 and UDP96008 in 'Dayezhugan' japanese apricot showed that SSRs obtained included a microsatellite locus originally identified in peach. The microsatellite sequence homogeneity between UDP96008 in japanese apricot in this study and UDP96008 in the peach in GenBank was $98 \%$. Twenty-four japanese apricot genotypes originating in diverse geographic areas had been identified with 14 SSR primer pairs developed in different species of Prunus. In total, 129 alleles were obtained and per primer pairs detected 2.5 alleles on the average. The results from cluster analysis showed that the genetic distance between 'Nanhong' and 'Zhonghong' was the closest, and cultivars from China and from Japan could not be separated completely.
\end{abstract}

Japanese apricot (Prunus mume Sieb. et Zucc.) originates in southern China and has been widely cultivated in China and Japan. It is used as fruit production and ornamental appreciation. Since most of the fresh Prunus mume fruit are sour, they are usually processed into different products, not for fresh-market sale, such as mume wine and candies. Several Prunus mume genotypes originating in China have been identified and classified with morphological characteristics (Gao et al., 1999a;Zhang et al., 1996) and isozyme analysis (Chu et al., 1988). However, these methods are limited with environmental influences and low levels of available polymorphism. The molecular markers, such as random fragment-length polymorphisms (RFLPs), randomly amplified polymorphic DNA (RAPD) markers, amplified fragment-length polymorphisms (AFLPs), and simple-sequence repeats (SSRs), open a new approach to characterize and study the genetic relationship among different species and cultivars. The SSR marker is generally codominant and highly polymorphic, and can be detected with a simple polymerase chain reaction (PCR) procedure and can be found in large numbers covering the whole genome of the plant. Prior studies have used RAPD markers to analyze parentage determination, classification and self-compatibility of japanese apricot (Ozaki et al., 1994; Ozaki and Shimada, 1995; Tao et al., 2000).

Received for publication 31 Oct. 2003. Accepted for publication 5 Apr. 2004. This research was supported in part by grants D/3232-1 from International Foundation for Science and 30070530 from National Natural Science Foundation of China.

${ }^{1}$ To whom reprint requests should be addressed; e-mail zhangzhen_nj@hotmail.com.
SSR marker is a powerful DNA marker to study molecular characterization of fruit trees (Gao et al., 2002b). Many studies on Prunus species demonstrate that primers developed in one species can amplify the microsatellite loci of some genotypes of other species (Cipriani et al., 1999; Downey and Iezzoni, 2000; Hormaza, 2002; Serrano et al., 2002). However, no application of SSR technique has been reported in japanese apricot, and no SSR primer pairs have been developed from japanese apricot so far.

In this study, we tried to use SSR primer pairs previously developed in other Prunus species to identify japanese apricot genotypes originating from diverse geographical regions and further to analyze their genetic relationship.

\section{Materials and Methods}

Plant material. In total, 24 japanese apricot genotypes (19 from China, 5 from Japan) were used in this study (Table 1). A young leaf tissue sample of each genotype was collected from the japanese apricot orchards located at Nanjing Agricultural University, Nanjing, China.

DNA extraction. Extracted DNA was qualified in a $1.0 \%$ agarose gel to check its completeness and then quantified spectrophotometrically (GeneQuant, Pharmacia Biotech, Piscataway, N.J.), diluted to $60 \mathrm{ng} \cdot \mu \mathrm{L}^{-1}$ with TE buffer and used for PCR amplification.

DNA amplification and sequencing of the SSR fragments. Japanese apricot microsatellites were PCR-amplified as previously described (Gao et al., 2002b). PCR reactions were carried out in a PTC-100 thermocycler (MJ-Research, Watertown, Mass.) by an initial denaturation for $8 \mathrm{~min}$ at $94{ }^{\circ} \mathrm{C}$ followed by one cycle of 2 min at $94{ }^{\circ} \mathrm{C}, 50 \mathrm{~s}$ at $61^{\circ} \mathrm{C}, 50 \mathrm{~s}$ at $72{ }^{\circ} \mathrm{C}$; then 20 cycles of $40 \mathrm{~s}$ at $94{ }^{\circ} \mathrm{C}, 50 \mathrm{~s}$ at $61{ }^{\circ} \mathrm{C}, 50 \mathrm{~s}$ at $72{ }^{\circ} \mathrm{C}$; and then 20 cycles of $45 \mathrm{~s}$ at $94^{\circ} \mathrm{C}, 50 \mathrm{~s}$ at $61^{\circ} \mathrm{C}, 50 \mathrm{~s}$ at $72{ }^{\circ} \mathrm{C}$; and a final extension of $7 \mathrm{~min}$ at $72{ }^{\circ} \mathrm{C}$. The PCR products were detected by a $8 \%$ nondenatured polyacrylamide gel electrophoresis in $1 \times \mathrm{TBE}$ buffer. Gels were run for $5 \mathrm{~h}$ at $250 \mathrm{v}$. Following the electrophoresis, the gel was silver-stained according to the protocol described by Gao et al. (2002b). The sizes of DNA fragments were estimated with the 50-bp ladder-DNA sizing markers (MBI Fermentas).

The amplification products produced by two SSR primer pairs (pchgmg2 and UDP96008) were sequenced and analyzed.

SSR of the cultivars and data analysis. Fourteen primer pairs as shown in Table 2

Table 1. List of the 24 japanese apricot cultivars used in this study and total number of DNA fragments.

\begin{tabular}{lcccc}
\hline $\begin{array}{l}\text { Code of } \\
\text { cultivars }\end{array}$ & Cultivars & $\begin{array}{c}\text { Local } \\
\text { origin }\end{array}$ & $\begin{array}{c}\text { Avg fruit } \\
\text { wt (g) }\end{array}$ & $\begin{array}{c}\text { Total no. } \\
\text { of bands }\end{array}$ \\
\hline 1 & Zaohong & Zhejiang (China) & 25.2 & 29 \\
2 & Zhonghong & Zhejiang (China) & 19.7 & 39 \\
3 & Wanhong & Zhejiang (China) & 15.6 & 35 \\
4 & Nanhong & Jiangsu (China) & 15.6 & 38 \\
5 & Xiaoyezhugan & Zhejiang (China) & 18.7 & 37 \\
6 & Dayezhugan & Zhejiang (China) & 20.8 & 34 \\
7 & Jiuzhongmei & Zhejiang (China) & 25.8 & 39 \\
8 & Longyanmei & Fujian (China) & 15.4 & 39 \\
9 & Yeliqing & Zhejiang (China) & 18.2 & 32 \\
10 & Henghe & Guangdong (China) & 32.2 & 39 \\
11 & Dalizhong & Guangdong (China) & 30.0 & 40 \\
12 & Zaohua & Guangdong (China) & 10.0 & 40 \\
13 & Tonglv & Hunan (China) & 12.3 & 28 \\
14 & Guangdonghuangpi & Guangdong (China) & 12.6 & 31 \\
15 & Siyuemei & Hunan (China) & 18.9 & 22 \\
16 & Sichuanhuangmei & Sichuan (China) & 20.0 & 32 \\
17 & Taoxingmei & Guangdong (China) & 18.2 & 40 \\
18 & Yunnanxingmei & Yunnan (China) & 29.0 & 35 \\
19 & Qijiangxingmei & Sichuan (China) & 22.4 & 35 \\
20 & Nankouume & Japan & 17.8 & 32 \\
21 & Shirokaga & Japan & 16.7 & 4.5 \\
23 & Hanakami & Japan & 15.2 & \\
\hline
\end{tabular}




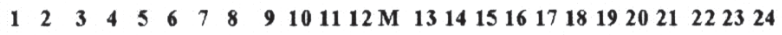

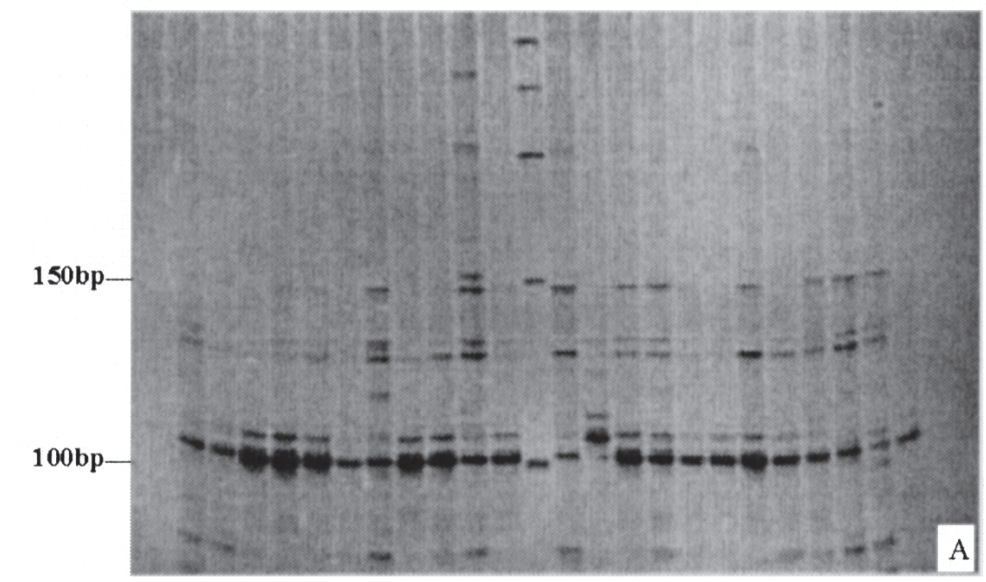

$123456789101112 M 131415161718192021222324$

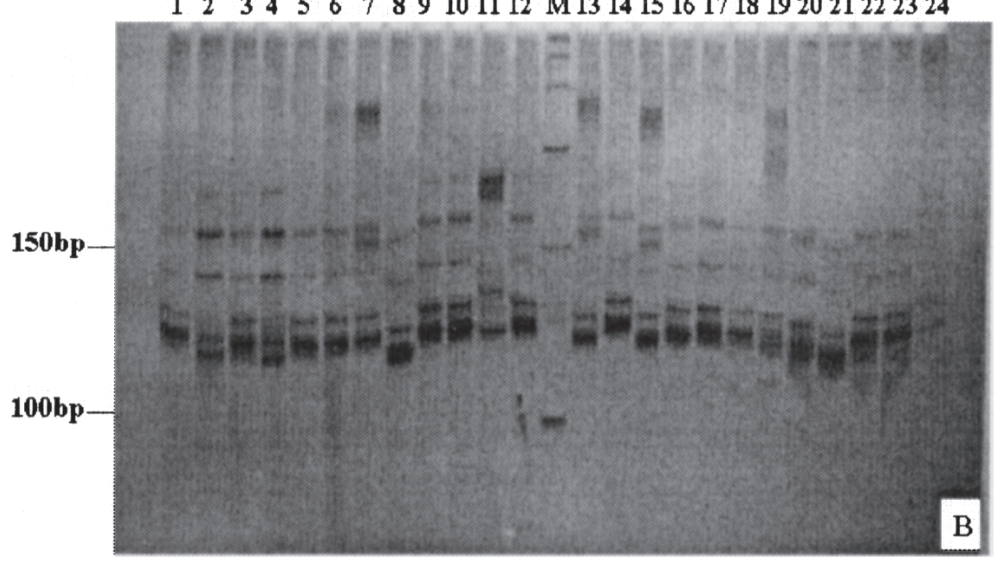

$\begin{array}{lllllllllllllllll}1 & 2 & 3 & 4 & 5 & 6 & 7 & 8 & 9 & 1011 & 12 & M 13 & 14 & 1516 & 17181920212223\end{array}$ 24

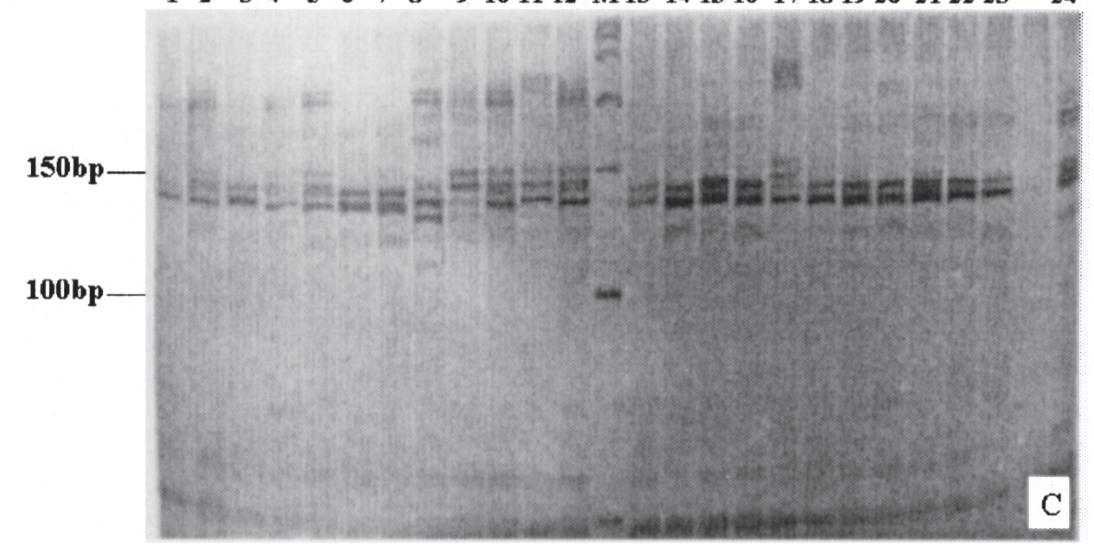

$\begin{array}{lllllllllllll}1 & 2 & 3 & 4 & 5 & 6 & 7 & 89 & 1011 & 12 M 131415161718192021222324\end{array}$

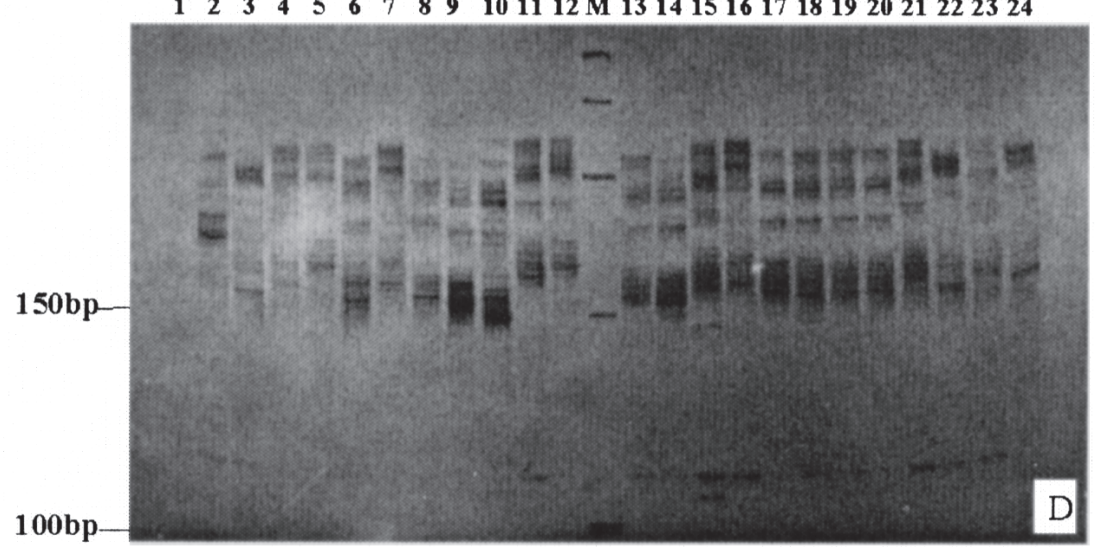

previously developed in peach and cherry (Cipriani et al., 1999; Sosinski et al., 2000) were used to identify the genetic diversity of 24 japanese apricot germplasm. Amplified fragments were scored as present or absent. A similarity matrix was generated using the equation discussed in Nei and $\mathrm{Li}$ (1979) based on the proportion of the shared amplification fragments. Genetic relationships among the 24 genotypes included in this study were investigated using unweighted pair group method with arithmetic mean (UPGMA) (Sokal and Michener, 1958) cluster analysis and depicted in a dendrogram. All analyses were computed with the SAS version 6.0 program.

\section{Results}

SSR analysis of japanese apricot germplasm. Of the 24 SSR primer pairs screened, three primer pairs (PS7a2, UDP97401and PS9f8) failed to amplify DNA bands in japanese apricot. Seven primer pairs failed to amplify DNA bands in all cultivars (UDP96015 and UDP97403) or the products were smeared (UDP96013, UDP97402, and UDP98406) or not repeatable (UDP98408 and Ps12e2). In total, 14 primer pairs were used in the analysis. From those primer pairs, 11 have been developed in peach, 1 in sour cherry and 2 in sweet cherry. The 14 primer pairs produced 129 alleles with a mean value of 2.5 alleles per locus. The size of the amplified fragments was consistent with that of expected from other species as shown in Table 2. The number of amplification bands per genotype was from 18 to 42 , and the average value was 35 as shown in Table 1. More than one polymorphic fragments were produced by all primer pairs except the primer pair PS8e8. Amplification patterns with primer pairs UDP98405, UDP96008, UDP98409 and pchgms 3 were as shown in Fig. 1. The frequency of DNA polymorphisms by SSR assay was sufficiently high to survey the phylogeny of Prunus mume. Six cultivars (Nos. $1,5,8,9,11,17)$ could be identified with the primer pairs UDP98409 as shown in Fig. 1C. Five cultivars (Nos. 8, 11, 13, 14, 23) could be identified with the primer pairs UDP98405 as shown in Fig. 1A, and no unique SSR primer pair could identify all cultivars.

The dendrogram was obtained after UPGMA cluster analysis of the similarity data as shown in Fig. 2. Genetic distance values between cultivars ranged from 0.39 to 1.10 . These cultivars were divided into two groups at 1.1 genetic distance. The value of genetic distance between cultivars 'Nanhong'and 'Zhonghong' was the smallest, which suggested that the genetic relationship of these two cultivars was the closest. The Dendrogram also showed that cultivars from China and from Japan could not be separated completely. Five cultivars from China ('Zaohong', 'Xiaoye-

Fig. 1. SSR banding patterns of 24 japanese apricot cultivars in polyacrylamide gel separation with different primer pairs: A UDP98405 B UDP96008 CUDP98409 D pchgms3 Lanes corresponding to the codes of the cultivars in Table 1. $\mathrm{M}=$ molecular marker (50-bp ladder). 
Table 2. List of SSR markers that produced polymorphic fragments among 24 japanese apricot cultivars.

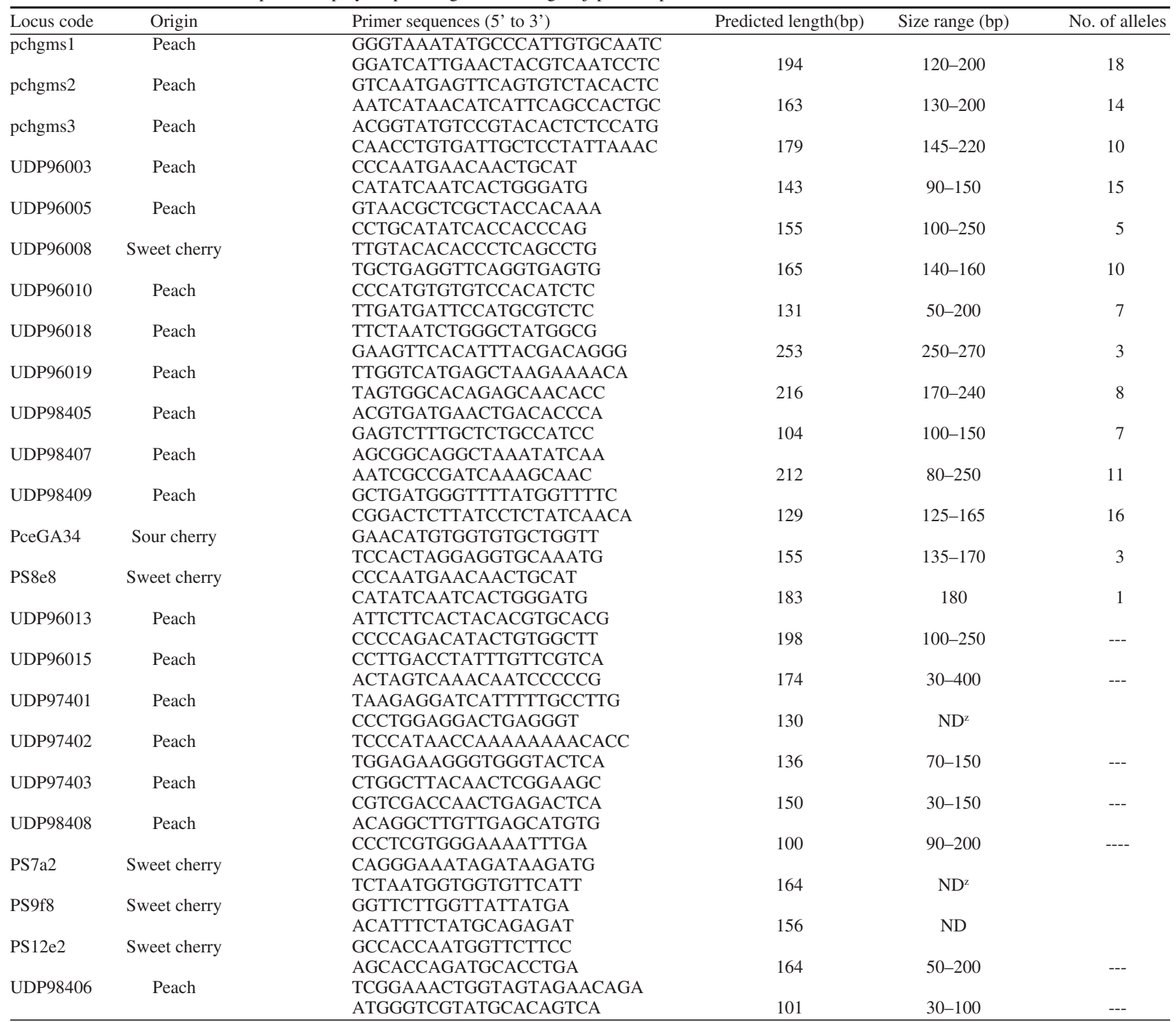

${ }^{2}$ No data.

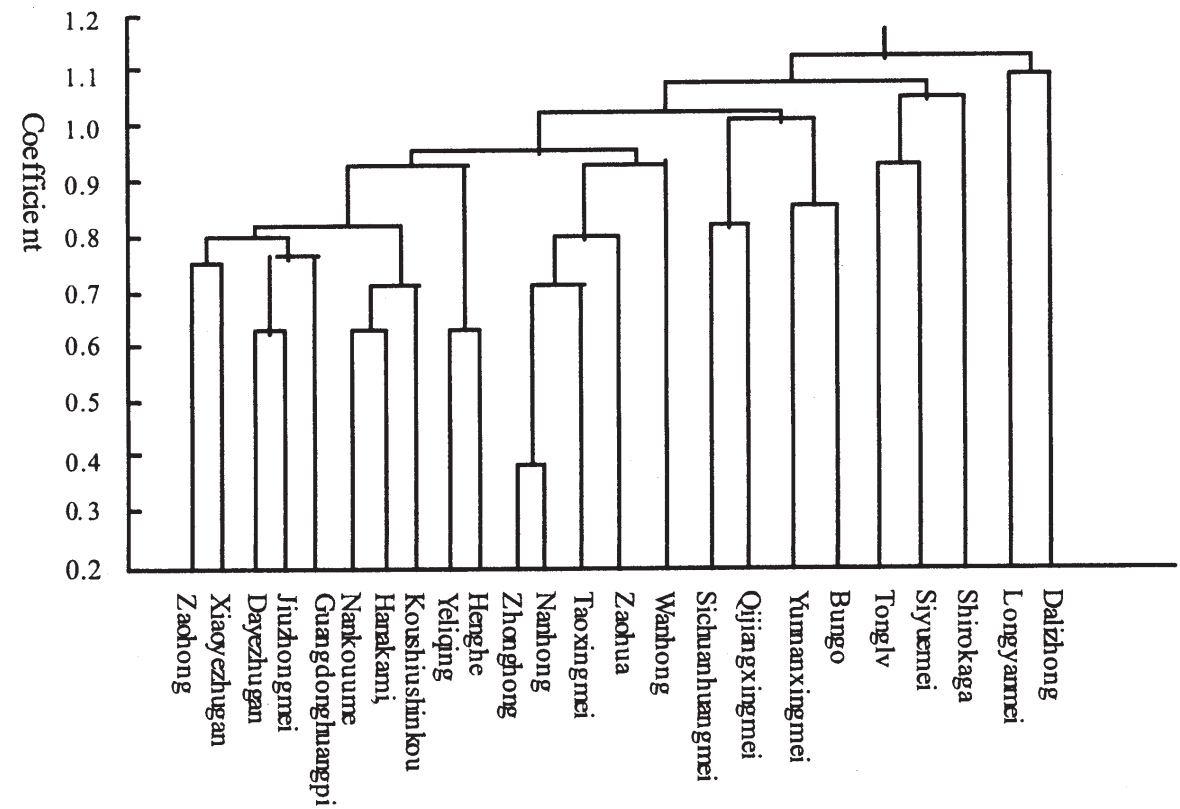

Fig. 2. Dendrogram of 24 japanese apricot cultivars based on UPGMA analysis using the similarity matrix generated by the Nei and Li coefficient after amplification with 14 pairs of microsatellite primers. 
(1)TTG TACA CA C CCTCAGCCTG TCACGCAAAA AGAAATAATA AGCAAGGGAG CA

(2) TTG TACA CAC CCTCAGCCTG TCACGCAAAA AGAATAATA AGCAAGGG CA

\section{(1)CCCAGG CA GG CATGATAA CACCTACACA CACACACACA ....GAG...AAACAGA}

\section{(2)CCCAGCA GGCATGATA CACCTACACA CA CACACACA CA GAGAG AAA CA GA}

\section{(1) GATAGTCAAC AACA CTCACC TGAaC CTCAG CA}

\section{(2)GATAGT CAACAACACT CACCTGAACC TCAGCA}

Fig. 3. Sequence alignment of UDP96008 SSR marker in different Prunus species. 1) sequence of UDP96008 from sweet cherry (GenBank accession No. AF501740). 2) sequence of PCR product from 'Dayezhugan' japanese apricot with UDP96008 primer pairs.

zhugan', 'Dayezhugan', 'Jiuzhongmei', 'Guangdonghuangpi') and three cultivars from Japan ('Nankouume', 'Hanakami', 'Koushiushinkou') were in one group at 0.82 genetic distance. Another group included two cultivars from China ('Tonglv', 'Siyuemei' and one cultivar from Japan 'Shirokaga').

Sequences of PCR products with SSR primer pairs pchgms 2 and UDP96008. The sequence of PCR products in japanese apricot cultivar Dayezhugan with SSR primer pairs pchgms2 was 138 base pairs, and the simple sequence repeat was $(\mathrm{AG})_{10}$, the following was the sequence of the microsatellite locus: CAGTTACTCA AGTCACAGAT GTGAGTTTTC TTGAACATTC TGAGTTTTGA ACTATCAGCCGAGATCAGAG AGAGAGAGAG AGAGAGAAGG GGGTTTTTTA ATGTAACGAT AAAAATCATA ACATCATTCA GCCACTGC (GenBank accession No. AY216737). The simple sequence repeat from peach is $(\mathrm{AG})_{24}$.

As for primer pair UDP96008, the simple sequence repeat obtained in japanese apricot was $(\mathrm{CA})_{8}$, whereas the simple sequence repeat obtained in sweet cherry was reported as (CA) ${ }_{7}$ (GenBank accession No.AF501740). The sequence homogeneity between the two microsatellites was $98 \%$ as shown in Fig. 3 .

\section{Discussion}

Sharing of SSR primer pairs between different species. Widespread use of SSR markers is often limited by the cost of developing of the primer pairs since it involves library generation and sequencing. Many studies indicate that microsatellite sequences are highly conserved in Prunus (Hormaza, 2002; Serrano et al., 2002). Recently SSR primer pairs have been developed from Prunus species, mostly peach (Aranzana et al., 2002; Cipriani et al., 1999; Dirlewanger et al., 2002; Sosinski et al., 2000; Testolin et al.; 2000), sweet cherry, sour cherry (Downey and Iezzoni, 2000), and apricot (Lopes et al., 2002). Though there are no SSR primers in japanese apricot, other Prunus SSR primer pairs could be used to identify japanese apricot germplasm. In this study, SSR amplification products in japanese apricot were sequenced and compared those obtained from sweet cherry. The result showed that the sequences obtained contained simple sequence repeats locus and the number of sequence repeats was different in different species. This study also demonstrated that SSR primer pairs developed in peach, sweet cherry and sour cherry could be shared in identification of japanese apricot germplasm.

Relationship among japanese apricot genotypes. Many researchers reported that Prunus mume is native to China and Prunus mume cultivars in Japan are introduced from China (Yoshida et al., 1999). In this study, cultivars from Japan could not be separated by SSR assay from cultivars from China, which suggested that there were no clear genome differences between Chinese cultivars and Japanese cultivars.

The genetic distance between cultivars 'Nanhong' and 'Zhonghong' was the closest from the dendrogram, which indicated that these two cultivars may have the same origin. The cluster results from SSR markers were in agreement with the results from morphological characteristics (Zhang et al., 1996; Gao et al., 1999a), which suggested that SSR marker could be used to study the genetic relationship of Prunus mume.

\section{Literature Cited}

Aranzana, M.J., J. Garcia-Mas, J. Carbo, and P. Arus. 2002. Development and variability analysis of microsatellite markers in peach. Plant Breeding 121:87-92

Cipriani, G., G. Lot, W.G. Huang, M. Marrazzo, E. Peterlunger, and R. Testolin. 1999. AC/GT and AG/CT microsatellite repeats in peach (Prunus persica (L) Batsch): Isolation, characterization and cross-species amplification in Prunus. Theor. Appl. Genet. 99:65-72.

Chu, M.Y., J. Ban, D.R. Lei, P. Chen, and A.M. Hua. 1988. A preliminary study on classification of japanese apricot (Prunus mume Sieb.etZucc.) by peroxidase isozyme. Fruit Sci. 5(2):62-64

Chu, M.X., J.Z. Wang, A.G. Wang, N. Li, and J.L. Fu. 2002. Cloning and sequencing of four microsatellite loci in Small Tail Han Yang sheep. Acta Genet. Sinica 29(5):402-405
Dirlewanger, E., P. Cosson, M. Tavaud, M.J. Aranzaza, C. Poizat, A. Zanetto, P. Arus, and F. Laigret. 2002. Development of microsatellite markers in peach (Prunus persica(L.) Batsch) and their use in genetic diversity analysis in peach and sweet cherry (Prunus avium L.). Theor. Appl. Genet.105:127-138

Downey, S.L. and A.F. Iezzoni. 2000. Polymorphic DNA markers in black cherry (Prunus serotina) are identified using sequences from sweet cherry, peach, and sour cherry. J. Amer. Soc. Hort. Sci. 125(1):76-80

Gao, Z.H., Z. Zhang, B.C. Sheng, and Y.M. Zhang. 1999a. Study on numerical taxonomy of fruiting mei (Prunus mume Sieb.et Zucc.) cultivars. J. Beijing For. Univ. 21(2):12-15

Gao, Z.H., Z. Zhang, and Z.H. Han. 2002a. SSR technique and development of the study in fruit science. J. Fruit Sci. 19(5):281-285

Gao, Z.H., Z. Zhang, B.C. Sheng, and Q.H. Yao. 1999b. Study on random amplified polymorphic DNA technique of Prunus mume. ZhiWu ShengLiXue TongXun 35(3):214-217

Gao,Z.H.,Z.Zhang,Z.H.Han, and Z.J. Shen. 2002b. Optimization on SSR analysis system of japanese apricot (Prunus mume Sieb. et Zucc). J. Nanjing Agr. Univ. 25(4):19-22

Hormaza, J.I. 2002. Molecular characterization and similarity relationships among apricot (Prunus armeniaca) genotypes using simple sequence repeats. Theor. Appl. Genet. 104:321-328

Lopes, M.S., K.M. Sefc, M. Laimer, and A.D. Machado. 2002. Identification of microsatellite loci in apricot. Mol. Ecol. Notes 2:24-26

Nei, M. and W.H. Li 1979. Mathematical model for studying genetic variation in terms of restriction endonucleases. Proc Natl Acad Sci USA 76:5269-5273

Ozaki T. and T. Shimada. 1995. RAPD analysis for parentage determination in Prunus mume Sieb.et Zucc. J. Jpn. Soc. Hort. Sci. 64(2):235-242

Ozaki T., T. Shimada, T. Nakanishi, J. Yaamamoto, and M. Yoshida. 1994. Classification of mume (Prunus mume Sieb. et Zucc) by RAPD assay. J. Jpn. Soc. Hort. Sci. 63(3):543-551

Serrano B.B., J. Gomez-Aparisi, and J.I. Hormaza. 2002. Molecular fingerprinting of Prunus rootstocks using SSRs. J. Hort. Sci. Biol. 77(3):368-372

Sokal R. and C. Michener. 1958. A statistical method for evaluating statistical relationships. Univ. Kan. Sci. Bul. 38:1409-1438

Sosinski B., M. Gannavarapu, L.D.Hager,L.E.Beck, G.J.King, C.D.Ryder, S.Rajapakse, W.V. Baird, R.E. Ballard, and A.G. Abbott. 2000. Characterization of microsatellite markers in peach [Prunus persica(L.) Baatsch]. Theor. Appl. Genet. 101:421-428

Tao, R., T. Habu, H. Yamane, A. Sugiura, and K. Iwamoto. 2000. Molecular Markers for selfcompatibility in japanese apricot (Prunus тите). HortScience 35(6):1121-1123

Testolin, R., T.Marrazzo, G. Cipriani, R. Quarta, I. Verde, M.T. Dettori, M. Pancaldi, and S. Sansavini. 2000. Microsatellite DNA in peach [Prunus persica $(\mathrm{L}$.) Batsch] and its use in fingerprinting and testing the genetic origin of cultivars. Genome 43:512-520

Zhang, Z., B.H. Cai, and C. Zhang. 1996. Classification of japanese apricot (Prunus mume Sieb.et Zucc.) based on morphological characteristics. Deciduous Fruits (3):35-36

Yoshida, M., N. Fujishige, and M. Yamaguchi. 1999. Fruit mume breeding in Japan. J. Beijing For. Univ. 21(2):37-41 